\section{Environmental Strategies to Promote Appropriate Behaviors and Social Interaction in Young Children}

\author{
Küçük Çocuklarda Uygun Davranışları ve \\ Sosyal Etkileşimi Desteklemek İçin \\ Çevresel Stratejiler
}

Turkish Journal of Special Education

Research and Practice

2021, Volume 3, Number 1, p 113-120 https://dergipark.org.tr/trsped

DOI: 10.37233/TRSPED.2021.0115

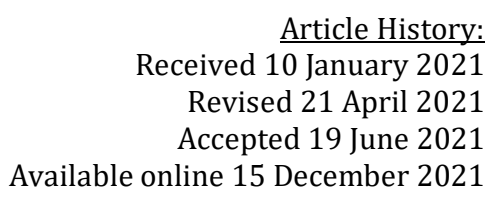

Article History:

ceived 10 January 2021

April 2021

Available online 15 December 2021

\author{
Şerife Balıkcı 1 1,2
}

\begin{abstract}
Executive Summary
Research suggests an increase in challenging behaviors among young children in early care and education settings (Granja et al., 2018). Challenging behaviors in early years are among the strongest predictors of more serious problem behaviors later in life (Brennan et al., 2015). High-quality, safe, responsive, and nurturing early care and education environments are critical to support development, engagement, and learning of young children with and without disabilities. Well-organized and engaging classroom environments help to prevent or decrease rates of challenging behaviors of young children by promoting appropriate behaviors and social interactions (Powell et al., 2006). Unfortunately, many practitioners working in early care and education settings are unsure how to create environments that support children's engagement and prevent challenging behaviors they exhibit (Steed and Roach, 2017). This practice brief offers recommendations and strategies for practitioner to design more engaging and developmentally appropriate classroom environments to support appropriate behaviors and prevent challenging behaviors of young children.
\end{abstract}

Keywords: Social development, appropriate behaviors, environmental strategies, young children.

\begin{abstract}
Özet
Araştırmalar, erken çocukluk bakım ve eğitim ortamlarında küçük çocuklar arasında zorlayıcı ve problem davranışlarda bir artış olduğunu göstermektedir (Granja vd., 2018). Erken çocukluk döneminde ortaya çıkan ve önlenmeyen zorlayıcı davranışlar, ilerleyen yaşlarda ortaya çıkan daha ciddi problem davranışlarının en güçlü yordayıcıları arasındadır (Brennan vd., 2015). Yüksek kaliteli, güvenli, duyarlı ve besleyici erken bakım ve eğitim ortamları, özel gereksinimi olan ya da normal gelişim gösteren küçük çocukların gelişimlerini, katılım davranışlarını ve öğrenmelerini desteklemek için çok önemlidir. İyi organize edilmiş ve ilgi çekici sınıf ortamları, uygun davranışları ve sosyal etkileşimleri teşvik ederek küçük çocukların zorlayıcı davranışlarını önlemeye veya azaltmaya yardımcı olur (Powell vd., 2006). Ne yazık ki, erken bakım ve eğitim ortamlarında çalışan birçok uygulamacı, çocukların aktif katılımlarını destekleyen ve sergiledikleri problem davranışları önleyen ortamları nasıl yaratacaklarından emin değiller (Steed ve Roach, 2017). Bu uygulama özeti, uygulamacılara, uygun davranışları desteklemek ve küçük çocukların zorlayıcı davranışlarını önlemek için daha ilgi çekici ve gelişimsel olarak uygun sınıf ortamları tasarlamaları için öneriler ve stratejiler sunmaktadır.
\end{abstract}

Anahtar Sözcükler: Sosyal gelişim, uygun davranışlar, çevresel stratejiler, küçük çocuklar.

Önerilen Atıf Şekli (Suggested Citation): Balıkcı, Ş. (2021). Environmental strategies to promote appropriate behaviors and social interaction in young children. Turkish Journal of Special Education Research and Practice, 3 (1), 113-120. https://doi.org/10.37233/TRSPED.2020.0115

\footnotetext{
${ }^{1}$ University of North Carolina Greensboro, Department of Specialized Education Services, School of Education, Greensboro, North

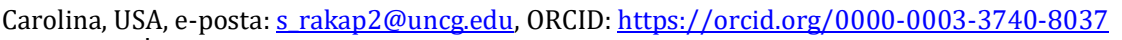

${ }^{2}$ Çarşamba İlçe Milli Eğitim Müdürlüğü, Samsun, Türkiye, e-posta: s rakap2@uncg.edu ORCID: https://orcid.org/0000-0003-3740$\underline{8037}$
} 


\section{Aim \& Background}

Improving positive outcomes for children with disabilities and their families is a central goal of early intervention services (Mitchell and Hauser-Cram, 2009). Described as "any repeated pattern of behavior that interferes with or is at risk of interfering with the child's optimal learning or engagement in pro-social interactions with peers and adults" (Smith and Fox, 2003), problem or challenging behaviors may undermine these positive child and family outcomes. Research has shown that the occurrence of challenging or problem behaviors in young children has been increasing (Granja et al., 2018) and young children with disabilities are more likely to exhibit these problems than children without disabilities (Mitchell and Hauser-Cram, 2009). Research has shown that unprevented challenging behaviors exhibited during early years have significant negative impact on children's readiness and adjustment to school (Kaiser, 2007) and other outcomes later in life (Miller et al, 2017; Powell et al, 2006). Moreover, studies reported that challenging behavior exhibited by young children with disabilities is addressed insufficiently by early care providers (Hemmeter et al., 2016). Practitioners working with young children should use effective practices to prevent challenging behaviors and promote social interactions of young children with disabilities. Environmental strategies have been found effective in preventing and reducing challenging behaviors and supporting social interactions of young children disabilities.

\section{Findings, Implications \& Recommendations}

Research has shown that carefully and effectively designed and maintained classroom environments can significantly increase positive social interactions among young children and prevent challenging behaviors they exhibit (Bovey and Strain, 2003; McWilliam and Bailey, 1995). Environmental strategies include changes and adaptations intentionally made to physical environment of a classroom setting (Division of Early Childhood, 2014). Although environmental strategies require planning and organization time outside of the instructional time in the classroom, they require little or no adult attention or involvement during implementation (Bovey and Strain, 2003). There are several environmental strategies that researchers suggest promoting positive social interactions and prevent challenging behaviors in early childhood classrooms. In this paper, four of these strategies are described: (a) using ability grouping, (b) using a balanced schedule of activities, and (c) using interesting and engaging classroom materials, and (d) arranging learning centers to support appropriate child behaviors.

\section{Strategy 1: Use of Ability Grouping}

When planning small group activities, have children who are socially more competent in the same group with children who are socially less skilled. Being in the same group or near to socially competent peers can help increase positive interactions and prevent challenging behaviors of young children (McCabe et al., 1999). However, providing access to a peer who is socially more skilled may not be sufficient to reach these goals. Teachers should intentionally support children's interaction by providing positive feedback to those who engage in positive social interactions and appropriate behaviors during activities (Hemmeter vd., 2020). Teachers should also observe children's positions during the activities so that children are faced towards each other and are able to see what the other child is doing (Hamilton, 2005). 


\section{Strategy 2: Use of a Balanced Schedule of Activities}

When creating your daily schedule, plan to have small and large group, teacher-directed and child-directed, active and quiet activities in balance. Large group, teacher-directed activities should be shorter than 20 min and include opportunities for active participation of young children (Devi et al., 2019). Teachers should only continue large group, teacherdirected activities when the majority of children are interested and engaged in the activity (Williams et al., 2019). Difficult activities should be placed at times children are more attentive. Teachers should have a schedule showing daily activities posted in the classroom. Posted schedule should be at children's eye level and include visual representation of each activity (Meadan et al., 2011). Teachers should review the schedule with children and prepare them when there is a change on the schedule.

\section{Strategy 3: Use Interesting and Engaging Classroom Materials}

When selecting materials and toys for children to play and use, have a variety of novel and familiar materials as well as toys that promote sharing and collaboration available. New materials in the environment can create opportunities for social interactions (Hemmeter et al., 2020). Teachers can rotate classroom toys, materials, or centers to give children a sense of newness. Materials or toys that allow or require children to play or work together also promote positive social interactions (Hamilton, 2005). Teacher should model expected behaviors (sharing or collaboration) for children who may need additional support. In addition, teachers should acknowledge and comment positively and descriptively on children who are playing together and sharing materials (Hemmeter et al., 2020).

\section{Strategy 4: Arrange Learning Centers Support Appropriate Child Behaviors}

When designing learning centers and activity areas, organize the spaces, furniture, materials, and toys to maximize appropriate behaviors for all children. Teachers should design areas with enough space for children to play by themselves or in small groups and establish clear boundaries to indicate where each learning area begins and ends (Gould and Sullivan, 1999). Teacher should also limit the number of learning centers available and the number of children who have access to a learning center at a given time. By limiting and rotating open learning centers and rotating limited number of children across centers, increase the changes for peer interactions by keeping the centers novel and interesting (Bovey and Strain, 2003).

\section{Conclusion}

Practitioners' use of environmental strategies results in increased social interactions among children and between children and adults in terms of its frequency and duration. As children become more engaged and show more appropriate behaviors, challenging behaviors is likely to decrease. High-quality early care and education environments serve as a long-term protecting factor for young children who are at risk for developing for more significant problem behaviors. 
Table 1. Environmental strategies resources for practitioners

\begin{tabular}{|c|c|}
\hline Resource and URL & Description \\
\hline $\begin{array}{l}\text { Division of Early Childhood Recommended Practices } \\
\text { https://www.dec-sped.org/dec-recommended- } \\
\text { practices }\end{array}$ & $\begin{array}{l}\text { Provides a list and description of DEC } \\
\text { Recommended Practices with respect } \\
\text { Environment strands and gives practical } \\
\text { examples. }\end{array}$ \\
\hline $\begin{array}{l}\text { IRIS Center } \\
\text { https://iris.peabody.vanderbilt.edu/module/env/\#c } \\
\text { ontent }\end{array}$ & $\begin{array}{l}\text { Includes a module with information practical } \\
\text { recommendations about early childhood } \\
\text { environments including designing physical } \\
\text { environment. }\end{array}$ \\
\hline $\begin{array}{l}\text { Early Childhood Technical Assistance Center } \\
\text { https://ectacenter.org/decrp/topic-environment.asp }\end{array}$ & $\begin{array}{l}\text { Offers resources (e.g., practice improvement } \\
\text { tools, environment checklists, environment } \\
\text { practice guides for practitioners and families) } \\
\text { about the DEC Recommended Practices } \\
\text { Environment strand. }\end{array}$ \\
\hline $\begin{array}{l}\text { National Center for Pyramid Model Innovations } \\
\text { https://challengingbehavior.cbcs.usf.edu/ }\end{array}$ & $\begin{array}{l}\text { Provides resources with respect to } \\
\text { implementation of Pyramid Model practices } \\
\text { which includes environmental strategies. }\end{array}$ \\
\hline $\begin{array}{l}\text { Center for Social and Emotional Foundations for Early } \\
\text { Learning } \\
\text { http://csefel.vanderbilt.edu/resources/training pres } \\
\text { chool.html }\end{array}$ & $\begin{array}{l}\text { Includes separate modules on creating } \\
\text { supportive environments for infants/toddlers, } \\
\text { preschool children, and resources for families. }\end{array}$ \\
\hline
\end{tabular}

\section{References}

Bovey, T., \& Strain, P. (2003). Using environmental strategies to promote positive social interactions (What Works Brief Series No. 6). Center on the Social and Emotional Foundations for Early Learning.

Brennan, L. M., Shaw, D. S., Dishion, T. J., \& Wilson, M. N. (2015). The predictive utility of early childhood disruptive behaviors for school-age social functioning. Journal of Abnormal Child Psychology, 43(6), 1187-1199. https://doi.org/10.1007/s10802$\underline{014-9967-5}$

Division for Early Childhood. (2014). DEC recommended practices in early intervention/early childhood special education 2014. Retrieved from http://www.decsped.org/dec-recommended-practices

Gould, P., \& Sullivan, J. (1999). The inclusive early childhood classroom: Easy ways to adapt learning centers for all children. Gryphon House, Inc.

Granja, M. R., Smith, S., Nguyen, U., \& Grifa, B. (2018). Learning about young children's challenging behavior and impacts on programs and families: A state-wide survey of Virginia's early care and education teachers. National Center for Children in Poverty, Mailman School of Public Health, Columbia University.

Hamilton, D. (2005). An ecobehavioural analysis of interactive engagement of children with developmental disabilities with their peers in inclusive preschools. International Journal of Disability, Development and Education, 52(2), 121-137. https://doi.org/10.1080/10349120500086363 
Hemmeter, M. L., Ostrosky, M., \& Fox, L. (2020). Unpacking the pyramid model: A practical guide for preschool teachers. Brookes Publishing.

Hemmeter, M. L., Snyder, P. A., Fox, L., \& Algina, J. (2016). Evaluating the implementation of the Pyramid Model for promoting social-emotional competence in early childhood classrooms. Topics in Early Childhood Special Education, 36(3), 133-146. https://doi.org/10.1177/0271121416653386

Hnatiuk, J. A., Salmon, J., Hinkley, T., Okely, A. D., \& Trost, S. (2014). A review of preschool children's physical activity and sedentary time using objective measures. American Journal of Preventive Medicine, 47(4), 487-497. https://doi.org/10.1016/j.amepre.2014.05.042

Kaiser, A. P. (2007). Addressing challenging behavior: Systematic problems, systematic solutions. Journal of Early Intervention, 29(2), 114-118. https://doi.org/10.1177/105381510702900204

McCabe, J. R., Jenkins, J. R., Mills, P. E., Dale, P. S., \& Cole, K. N. (1999). Effects of group composition, materials, and developmental level on play in preschool children with disabilities. Journal of Early Intervention, 22(2), 164-178. https://doi.org/10.1177/105381519902200208

McWilliam, R. A., \& Bailey Jr, D. B. (1995). Effects of classroom social structure and disability on engagement. Topics in Early Childhood Special Education, 15(2), 123147. https://doi.org/10.1177/027112149501500201

Meadan, H., Ostrosky, M. M., Triplett, B., Michna, A., \& Fettig, A. (2011). Using visual supports with young children with autism spectrum disorder. Teaching Exceptional Children, 43(6), 28-35. https://doi.org/10.1177/004005991104300603

Miller, S., Smith-Bonahue, T., \& Kemple, K. (2017). Preschool teachers' responses to challenging behavior: The role of organizational climate in referrals and expulsions. International Research in Early Childhood Education, 8(1), 38-57.

Mitchell, D. B., \& Hauser-Cram, P. (2009). Early predictors of behavior problems: Two years after early intervention. Journal of Early Intervention, 32(1), 3-16. https://doi.org/10.1177/1053815109349113

Powell, D., Dunlap, G., \& Fox, L. (2006). Prevention and intervention for the challenging behaviors of toddlers and preschoolers. Infants \& Young Children, 19(1), 25-35.

Smith, B., \& Fox, L. (2003). Systems of service delivery: A synthesis of evidence relevant to young children at risk of or who have challenging behavior. Center for Evidence-Based Practice: Young Children with Challenging Behavior, University of South Florida.

Steed, E. A., \& Roach, A. T. (2017). Childcare providers' use of practices to promote young children's social-emotional competence. Infants \& Young Children, 30(2), 162-171. https://doi.org/10.1097/IYC.0000000000000092

Williams, P., Sheridan, S., \& Pramling Samuelsson, I. (2019). A perspective of group size on children's conditions for wellbeing, learning and development in preschool. Scandinavian Journal of Educational Research, 63(5), 696-711. https://doi.org/10.1080/00313831.2018.1434823 


\section{Uygulama Özeti}

\section{Küçük Çocuklarda Uygun Davranışları ve Sosyal Etkileşimi Desteklemek İçin Çevresel Stratejiler}

\section{Amaç ve Kapsam}

Özel gereksinimli çocuklar ve aileleri için olumlu sonuçların elde edilmesi erken müdahale hizmetlerinin temel hedefidir (Mitchell ve Hauser-Cram, 2009). "Çocuğun öğrenmesine veya akranları ya da yetişkinlerle olan sosyal etkileșimlere aktif katılmasına engel olan ya da olma riski bulunan davranış kalıbı" (Smith ve Fox, 2003) olarak tanımlanan problem veya zorlayıcı davranışlar, erken müdahalenin çocuk ve aile çıktıları üzerindeki olası olumlu sonuçlarını zayıflatabilir. Araştırmalar, küçük çocuklarda görülen zorlayıcı veya problem davranışların sayı ve yoğunluğunun arttığını (Granja vd., 2018) ve özel gereksinimli küçük çocukların, normal gelişim gösteren akranlarına oranla bu sorunları sergileme olasılıklarının daha yüksek olduğunu göstermektedir (Mitchell ve Hauser-Cram, 2009). Araştırmalar, erken yaşlarda ortaya çıkan ve önlenmeyen zorlayıcı, problem davranışların, çocukların okula hazır olma halini ve uyumunu (Kaiser, 2007) ve ileriki yaşantılarındaki başarılarını (Miller vd., 2017; Powell vd., 2006) olumsuz yönde etkilediğini göstermektedir. Ayrıca araştırmalar, özel gereksinimli küçük çocukların sergilediği zorlu davranışların erken bakım ve eğitim sağlayan bakıcı, öğretmen ve diğer uzmanlar tarafından yetersiz bir şekilde ele alındığını göstermektedir (Hemmeter vd., 2016). Küçük çocuklarla çalışan uygulamacılar, zorlayıcı, problem davranışları önlemek ve özel gereksinimli küçük çocukların sosyal etkileşimlerini teşvik etmek için etkili uygulamaları kullanmalıdır. Çevresel stratejilerin zorlu davranışları önlemede ve azaltmada ve özel gereksinimli küçük çocukların sosyal etkileşimlerini desteklemede etkili olduğu bulunmuştur.

\section{Bulgular, Çıkarımlar ve Öneriler}

Araştırmalar, dikkatli ve etkili bir şekilde tasarlanmış ve sürdürülen sınıf ortamlarının küçük çocuklar arasındaki olumlu sosyal etkileşimleri önemli ölçüde artırabileceğini ve bu çocukların sergiledikleri zorlu davranışları önleyebileceğini göstermiştir (Bovey ve Strain, 2003; McWilliam ve Bailey, 1995). Çevresel stratejiler, bir sınıfın fiziksel ortamında amaçlı olarak yapılan değişiklikleri ve uyarlamaları içerir (Erken Çocukluk Birimi, 2014). Çevresel stratejiler, sınıftaki öğretim süresi dişında planlama ve organizasyon süresi gerektirse de uygulama sırasında çok az veya hiç yetişkin ilgisi veya katılımı gerektirmez (Bovey ve Strain, 2003). Araştırmacıların, erken çocukluk sınıflarında olumlu sosyal etkileşimleri teşvik etmek ve zorlayıcı davranışları önlemek için önerdiği birkaç çevresel strateji vardır. Bu uygulama özetinde bu stratejilerden dört tanesine yer verilmiştir: (a) yetenek gruplarının kullanılması, (b) dengeli bir etkinlik programı kullanılması ve (c) ilginç ve ilgi çekici sınıf materyallerinin kullanılması ve (d) uygun çocuk davranışlarını desteklemek için öğrenme merkezlerinin düzenlenmesi.

\section{Strateji 1: Yetenek Gruplarının Kullanılması}

Küçük grup aktivitelerini planlarken, sosyal olarak daha az yetenekli çocuklarla sosyal olarak daha yetkin olan çocukların aynı grupta yer aldığından emin olunmalıdır. Aynı grupta olmak veya sosyal açıdan yetkin akranlara yakın olmak, küçük çocukların olumlu 
etkileşimlerini artırmaya ve zorlu davranışları önlemeye yardımcı olabilir (McCabe vd., 1999). Bununla birlikte, sosyal olarak daha yetenekli bir akrana erişim sağlamak, bu hedeflere ulaşmak için yeterli olmayabilir. Öğretmenler, etkinlikler sırasında olumlu sosyal etkileşim ve uygun davranışlarda bulunanlara olumlu geri bildirim sağlayarak çocukların etkileşimini bilinçli olarak desteklemelidir (Hemmeter vd., 2020). Öğretmenler, ayrıca, etkinlikler sırasında çocukların pozisyonlarını gözlemleyerek yüzlerinin birbirlerine dönük olduğundan emin olmalıdır; bu bir çocuğun diğer çocuğun ne yaptığını görmesine olanak tanır (Hamilton, 2005).

\section{Strateji 2: Dengeli Bir Etkinlik Programı Kullanılması}

Günlük programınızı oluştururken, küçük ve büyük grup, öğretmen ve çocuk merkezli, aktif ve sessiz aktiviteler dengede olacak șekilde planlama yapmanız oldukça önemlidir. Öğretmen tarafından yönlendirilen büyük grup etkinlikleri 20 dakikadan kısa olmalı ve küçük çocukların aktif katılımı için fırsatlar içermelidir (Devi vd., 2019). Öğretmenler, yalnızca çocukların çoğunluğu etkinlikle ilgilendiğinde ve bu etkinlikle meşgul olduğunda öğretmen merkezli büyük grup etkinliklere devam etmelidir (Williams vd., 2019). Zor aktiviteler, çocukların daha ilgili ve uyanık olduğu zamanlarda yapılmalıdır. Öğretmenlerin sınıfta sergiledikleri, günlük aktiviteleri gösteren bir programı olmalıdır. Sınıfta sergilenen program çocukların göz hizasında olmalı ve her aktivitenin görsel sunumunu içermelidir (Meadan vd., 2011). Öğretmenler programı çocuklarla birlikte gözden geçirmeli ve programda bir değişiklik olduğunda onları bu değişiklik için önceden hazırlamalıdır.

\section{Strateji 3: İlginç ve İlgi Çekici Sınıf Materyallerinin Kullanılması}

Çocukların oynaması ve kullanması için malzeme ve oyuncak seçerken, çeşitli yeni ve tanıdık malzemelerin yanı sıra paylaşımı ve işbirliğini teşvik eden oyuncaklarında seçilmesi oldukça önemlidir. Ortamdaki yeni malzemeler sosyal etkileşimler için firsatlar yaratabilir (Hemmeter vd., 2020). Öğretmenler, çocuklara yenilik hissi vermek için sınıf oyuncaklarını, materyallerini veya merkezleri rotasyona sokabilirler. Çocukların birlikte oynamasına veya birlikte çalışmasına izin veren veya bunu gerektiren malzemeler veya oyuncaklar da olumlu sosyal etkileşimleri teşvik eder (Hamilton, 2005). Öğretmenler, ek desteğe ihtiyaç duyabilecek çocuklar için onlardan beklenen davranışları (paylaşım veya işbirliği) modellemelidir. Ayrıca öğretmenler birlikte oynayan ve materyalleri paylaşan çocukları olumlu ve betimleyici bir şekilde onaylamalı ve sınıfa duyurmalıdır (Hemmeter vd., 2020).

\section{Strateji 4: Uygun Çocuk Davranışlarını Desteklemek İçin Öğrenme Merkezlerinin Düzenlenmesi}

Öğrenme merkezleri ve aktivite alanları tasarlarken, tüm çocuklar için uygun davranışları en üst düzeye çıkarmak için alanları, mobilyaları, malzemeleri ve oyuncakları düzenlemek gerekmektedir. Öğretmenler, çocukların kendi başlarına veya küçük gruplar halinde oynayabileceği yeterli alana sahip ortamlar, etkinlik merkezleri tasarlamalı ve her bir öğrenme alanının nerede başladığını ve bittiğini belirtmek için net sınırlar oluşturmalıdır (Gould ve Sullivan, 1999). Öğretmenler, ayrıca mevcut öğrenme merkezlerinin sayısını ve belirli bir zamanda bir öğrenme merkezine erișimi olan çocukların sayısını sınırlamalıdır. Açık öğrenme merkezlerini sınırlandırarak ve sınırlı sayıda çocuğun merkezlerde oynamasına ve merkezler arası geçiş yapmasına izin vererek, öğrenme merkezleri yeni ve ilginç kılınabilir ve akran etkileşimlerini destekleyebilir (Bovey ve Strain, 2003). 


\section{Sonuç}

Yüksek kaliteli erken bakım ve eğitim ortamları, daha ciddi davranış problemleri geliştirme riski taşıyan küçük çocuklar için uzun vadeli bir koruyucu faktör görevi görür. Uygulamacıların çevresel stratejileri kullanması, çocuklar arasında ve çocuklar ile yetişkinler arasındaki sosyal etkileșimlerin sıklığının ve süresinin artmasına yardımcı olur. Çocuklar daha ilgili hale geldikçe ve daha uygun davranışlar gösterdikçe, sergiledikleri problem davranışlar azalacaktır.

Tablo 1. Uygulamacılar için çevresel strateji kaynakları

\begin{tabular}{|c|c|}
\hline Kaynak ve URL & Tanım \\
\hline $\begin{array}{l}\text { Erken Çocukluk Birimi Tavsiye Edilen Uygulamalar } \\
\text { https://www.dec-sped.org/dec-recommended- } \\
\text { practices }\end{array}$ & $\begin{array}{l}\text { Çevre ile ilgili Erken Çocukluk Birimi (DEC) } \\
\text { Tavsiye Edilen Uygulamaların bir listesini ve } \\
\text { açıklamasını sağlar ve pratik örnekler verir. }\end{array}$ \\
\hline $\begin{array}{l}\text { IRIS Merkezi } \\
\text { https://iris.peabody.vanderbilt.edu/module/env/\#c } \\
\text { ontent }\end{array}$ & $\begin{array}{l}\text { Fiziksel çevrenin tasarlanması da dahil olmak } \\
\text { üzere erken çocukluk ortamları hakkında pratik } \\
\text { bilgiler içeren bir modül içerir. }\end{array}$ \\
\hline $\begin{array}{l}\text { Erken Çocukluk Teknik Destek Merkezi } \\
\text { https://ectacenter.org/decrp/topic-environment.asp }\end{array}$ & $\begin{array}{l}\text { DEC Tavsiye Edilen Uygulamalardan Ortam ile } \\
\text { ilgili kaynaklar (örn., uygulama geliştirme } \\
\text { araçları, çevre kontrol listeleri, uygulamacılar ve } \\
\text { aileler için çevre uygulama kılavuzları) sunar. }\end{array}$ \\
\hline $\begin{array}{l}\text { Ulusal Piramit Modeli Yenilikleri Merkezi } \\
\text { https://challengingbehavior.cbcs.usf.edu/ }\end{array}$ & $\begin{array}{l}\text { Çevresel stratejileri içeren Piramit Modeli } \\
\text { uygulamalarının kullanılmasına ilişkin kaynak } \\
\text { sağlar. }\end{array}$ \\
\hline $\begin{array}{l}\text { Erken Öğrenme için Sosyal ve Duygusal Temeller } \\
\text { Merkezi } \\
\text { http://csefel.vanderbilt.edu/resources/training pres } \\
\text { chool.html }\end{array}$ & $\begin{array}{l}\text { Bebekler, küçük çocuklar ve okul öncesi } \\
\text { çocukları için destekleyici ortamlar ve aileler } \\
\text { için kaynaklar oluşturmaya yönelik ayrı } \\
\text { modüller içerir. }\end{array}$ \\
\hline
\end{tabular}

\section{Yazar(lar)in Beyanı}

Araştırmacıların katkı oranı beyanı: Araştırmacılar çalışmaya eşit oranda katkı yapmışlardır.

Etik Kurul Kararı: Bu makalede sunulan çalışmanın bir uygulama özeti çalışması olması nedeniyle etik kurul iznine gerek duyulmamaktadır.

Çatışma beyanı: Araştırmada yazarlar arasında ya da diğer kişi/kurum/kuruluşlarla herhangi bir çıkar çatışması bulunmamaktadır.

Destek ve teşekkür: $\mathrm{Bu}$ araştırma için herhangi bir kurumdan finansal destek alınmamıştır. 